Article

\title{
Healthy Subcutaneous and Omental Adipose Tissue Is Associated with High Expression of Extracellular Matrix Components
}

\author{
Matúš Soták 1,2,3,+ , Meenu Rohini Rajan ${ }^{1,2,3,+}$, Madison Clark ${ }^{1,2}$, Christina Biörserud ${ }^{4,5}$, Ville Wallenius ${ }^{4,5} \mathbb{D}$, \\ Carolina E. Hagberg ${ }^{6,7}$ (D) and Emma Börgeson 1,2,3,*(D)
}

Citation: Soták, M.; Rajan, M.R.; Clark, M.; Biörserud, C.; Wallenius, V.; Hagberg, C.E.; Börgeson, E. Healthy Subcutaneous and Omental Adipose Tissue Is Associated with High Expression of Extracellular Matrix Components. Int. J. Mol. Sci. 2022, 23, 520. https://doi.org/ $10.3390 /$ ijms 23010520

Academic Editors: Nobuyuki

Takahashi and Ingrid

Wernstedt Asterholm

Received: 31 October 2021

Accepted: 30 December 2021

Published: 4 January 2022

Publisher's Note: MDPI stays neutral with regard to jurisdictional claims in published maps and institutional affiliations.

Copyright: (C) 2022 by the authors. Licensee MDPI, Basel, Switzerland. This article is an open access article distributed under the terms and conditions of the Creative Commons Attribution (CC BY) license (https:// creativecommons.org/licenses/by/ $4.0 /)$.
1 Institute of Medicine, Department of Molecular and Clinical Medicine, Wallenberg Laboratory, Sahlgrenska Academy, University of Gothenburg, 40530 Gothenburg, Sweden; matus.sotak@wlab.gu.se (M.S.); meenu.rajan@wlab.gu.se (M.R.R.); madison.borgesonlab@gmail.com (M.C.)

2 Wallenberg Centre for Molecular and Translational Medicine, Sahlgrenska Academy, University of Gothenburg, 40530 Gothenburg, Sweden

3 Region Vaestra Goetaland, Department of Clinical Physiology, Sahlgrenska University Hospital, 41345 Gothenburg, Sweden

4 Region Vaestra Goetaland, Department of Surgery, Sahlgrenska University Hospital, 41345 Gothenburg, Sweden; christina.biorserud@vgregion.se (C.B.); ville.wallenius@gastro.gu.se (V.W.)

5 Department of Surgery, Institute of Clinical Sciences, Sahlgrenska Academy, University of Gothenburg, 40530 Gothenburg, Sweden

6 Division of Cardiovascular Medicine, Department of Medicine Solna, Karolinska Institutet, 17164 Stockholm, Sweden; Carolina.Hagberg@ki.se

7 Center for Molecular Medicine, Karolinska Institutet, 17174 Stockholm, Sweden

* Correspondence: emma.borgeson@wlab.gu.se; Tel.: +46-31-3423833

+ These authors contributed equally to this work.

\begin{abstract}
Obesity is associated with extensive expansion and remodeling of the adipose tissue architecture, including its microenvironment and extracellular matrix (ECM). Although obesity has been reported to induce adipose tissue fibrosis, the composition of the ECM under healthy physiological conditions has remained underexplored and debated. Here, we used a combination of three established techniques (picrosirius red staining, a colorimetric hydroxyproline assay, and sensitive gene expression measurements) to evaluate the status of the ECM in metabolically healthy lean (MHL) and metabolically unhealthy obese (MUO) subjects. We investigated ECM deposition in the two major human adipose tissues, namely the omental and subcutaneous depots. Biopsies were obtained from the same anatomic region of respective individuals. We found robust ECM deposition in MHL subjects, which correlated with high expression of collagens and enzymes involved in ECM remodeling. In contrast, MUO individuals showed lower expression of ECM components but elevated levels of ECM cross-linking and adhesion proteins, e.g., lysyl oxidase and thrombospondin. Our data suggests that subcutaneous fat is more prone to express proteins involved in ECM remodeling than omental adipose tissues. We conclude that a more dynamic ability to deposit and remodel ECM may be a key signature of healthy adipose tissue, and that subcutaneous fat may adapt more readily to changing metabolic conditions than omental fat.
\end{abstract}

Keywords: adipose tissue; fibrosis; adipose tissue fibrosis; extracellular matrix; ECM remodeling; obesity; metabolic health; metabolically unhealthy obese; cardiometabolic disease

\section{Introduction}

White adipose tissue (WAT) is a highly regulated endocrine organ that plays a critical role in maintaining whole body energy homeostasis. WAT can be compartmentalized into two major anatomically distinct depots (subcutaneous and visceral WAT), further subdivided based on specific locations. Omental fat (oWAT) is the predominant visceral 
fat depot in humans, whereas the subcutaneous fat (scWAT) of the abdomen is the best studied. These two fat depots are often compared and have been shown to differ in their cellular composition and morphology, secretory profile, and metabolic characteristics [1]. Whereas scWAT is the primary site for lipid storage in healthy humans, oWAT is thought to mainly serve as an immunomodulatory cushion for the gastro-intestinal tract. These depot-specific functions are also reflected by differences in the tissue microenvironment, propensity to inflame, and metabolic regulation [2]. Obesity-induced accumulation of lipids in oWAT is associated with inflammation and a greatly increased risk of developing cardiometabolic diseases. In contrast, subcutaneous obesity may present a metabolic advantage and even reduce the risk for disease development [3-5]. Depending on their fat distribution and adipose tissue functionality, not all obese individuals develop comorbidities such as insulin resistance or hypertension. Likewise, not all lean individuals are free from metabolic disturbances, and may even display the metabolic characteristics associated with obesity. It has become apparent that more knowledge is needed about the pathologies associated with obesity and excessive fat accumulation. A better understanding of the morphological changes associated with diseases within each WAT depot would also allow a better categorization of patients based on metabolic state in the clinic, and enable more targeted development of future therapies within patient subgroups.

WAT is an organ with high plasticity, which can rapidly respond to alterations in energy status by undergoing extensive remodeling and expansion. The adipocytes are surrounded by an extracellular matrix (ECM) that forms a dense three-dimensional network of fibrous proteins e.g., collagens, adhesion proteins, proteoglycans, and modulatory enzymes such as matrix metalloproteinases (MMPs). These proteins provide structural integrity to the tissue and modulate several biological processes, such as cell adhesion, migration, proliferation and differentiation, and mediate signal transduction by sequestering and releasing various growth factors [6]. During weight-gain induced adipose tissue expansion, the ECM needs to be remodeled and reorganized to allow adipocytes to expand within the tissue. ECM accumulation depends on a balance between collagen synthesis and assembly (through the expression of components such as collagens and procollagen C-endopeptidase enhancer (PCOLCE)), and degradation of existing ECM (regulated by MMPs and their inhibitors, called tissue inhibitor of matrix metalloproteinases (TIMPs)) [7]. Healthy expansion of adipose tissue also requires adequate vascularization, regulated mainly by vascular endothelial growth factor A (VEGF-A), which in turn is induced by hypoxia [8]. Thus, an effective adipogenic and angiogenic response with appropriate ECM remodeling is required to maintain metabolically healthy adipose tissue during weight gain $[9,10]$. However, the question remains what characterizes the ECM of metabolically healthy lean (MHL) individuals compared with those of metabolically unhealthy obese (MUO) patients, and what differences can be found between human oWAT and scWAT fat depots.

Excessive and/or dysregulated WAT expansion triggers many pathological effects such as hypoxia, altered chemokine/adipokine secretion, and ECM deposition. Together, these changes lead to the development of chronic low-grade inflammation in WAT, which ultimately results in systemic metabolic disturbances. One of the long-term consequences of dysregulated WAT remodeling is the development of pathological ECM deposition, often referred to as fibrosis, which is believed to stem from the chronic unresolved inflammation that can develop due to unhealthy WAT expansion [11]. Fibrosis is characterized by excessive accumulation of certain ECM components and modulatory enzymes. The majority of the reports demonstrate high WAT fibrosis in human obesity [12-16], with oWAT being more prone to develop fibrosis than scWAT [12,17]. However, the subject remains controversial as several contradictory reports have been published [18-20]. A direct comparison of these studies may be difficult due to differences in the criteria and methodologies used for the assessment of the ECM status and fibrosis level in human fat biopsies. Moreover, the use of biopsies from different anatomical sites and mixed popula- 
tions of metabolically healthy and unhealthy obese subjects may add additional complexity when comparing reports.

To investigate the detailed quantity and composition of the adipose tissue ECM during health and disease, we studied paired oWAT and scWAT biopsies taken from the same anatomical locations of two distinctly different groups of subjects: MHL and MUO individuals. Using three complementary methods, we show that, at least for the subjects included in this study, MHL typically display more ECM deposition than MUO subjects. MHL also showed higher expression of transcripts associated with ECM remodeling, with the notable exception of lysyl-oxidase (LOX) and thrombospondin 1 (THBS1), which were higher in MUO. In addition, we found many ECM related transcripts to be expressed at higher levels in scWAT as compared with oWAT. Together, our results suggest that healthy adipose tissue is characterized by a higher flexibility that allows for rapid remodeling of the ECM, while the ECM surrounding adipose tissue in obese subjects may be stiffer and less adaptable to changing conditions.

\section{Results}

\subsection{Patient Characteristics}

We recruited two distinctly different patient groups of MHL and MUO individuals to quantify ECM deposition in human WAT biopsies. A metabolically unhealthy phenotype was defined as displaying three or more of the metabolic syndrome criteria described by the International Diabetes Federation [21] (see methods section for further details). Paired oWAT and scWAT samples were obtained from all participants. Furthermore, the adipose tissue from lean and obese subjects was obtained from the same anatomical subregion. Both patient groups underwent a detailed health exam and were characterized in the clinic by anthropometrical, clinical and biochemical measurements (Table 1). The groups did not differ significantly in age, and both groups included female and male participants, although the patient group size was not sufficiently large to determine the impact of sex. Compared with MHL, body weight, BMI, and waist circumference were higher in MUO, as expected.

Table 1. Patient characteristics. Data are presented as the median and interquartile range (25-75\%). The differences between metabolically healthy lean (MHL) and metabolically unhealthy obese (MUO) groups were assessed using Mann-Whitney $U$ test for numerical values, and Fisher's exact test for categorical variables. ${ }^{*} p \leq 0.05 ;{ }^{* *} p \leq 0.01 ;{ }^{* * *} p \leq 0.001$.

\begin{tabular}{|c|c|c|c|c|}
\hline Variables & MHL & MUO & $p$ Values & Significance \\
\hline \multicolumn{5}{|c|}{ Characteristics } \\
\hline$n$ & 8 & 15 & & \\
\hline Sex & 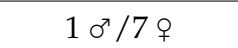 & 4 o $^{x} / 11$ 우 & & \\
\hline Age (years) & $40.0(33.8-47.2)$ & $47.0(35.0-54.0)$ & 0.583 & \\
\hline \multicolumn{5}{|l|}{ Anthropometrics } \\
\hline Body weight (kg) & $64.0(59.2-65.5)$ & $\begin{array}{c}131.5 \\
(113.8-138.0)\end{array}$ & 0.0001 & $* * *$ \\
\hline $\mathrm{BMI}\left(\mathrm{kg} / \mathrm{m}^{2}\right)$ & $22.2(21.7-23.2)$ & $43.2(39.6-44.9)$ & $<0.0001$ & $* * *$ \\
\hline Waist circumference (cm) & $80.0(77.5-88.0)$ & $\begin{array}{c}124.5 \\
(120.0-129.5)\end{array}$ & 0.0001 & $* * *$ \\
\hline \multicolumn{5}{|c|}{ Clinical measurements } \\
\hline BP diastolic (mm Hg) & $72.5(69.5-77.0)$ & $88.0(81.0-96.0)$ & 0.002 & $* *$ \\
\hline BP systolic (mm Hg) & $\begin{array}{c}119.0 \\
(113.5-123.5)\end{array}$ & $\begin{array}{c}139.0 \\
(132.5-148.5)\end{array}$ & 0.0009 & $* * *$ \\
\hline
\end{tabular}


Table 1. Cont.

\begin{tabular}{|c|c|c|c|c|}
\hline Variables & MHL & MUO & $p$ Values & Significance \\
\hline Heart Rate (bpm) & $79.5(76.5-81.8)$ & $83.0(69.5-89.5)$ & 0.815 & \\
\hline Triglycerides (mmol/L) & $0.8(0.7-0.9)$ & $1.2(1.0-1.6)$ & 0.024 & * \\
\hline Cholesterol (mmol/L) & $4.6(4.5-5.2)$ & $4.8(4.2-5.0)$ & 0.569 & \\
\hline $\begin{array}{l}\text { HDL-cholesterol } \\
(\mathrm{mmol} / \mathrm{L})\end{array}$ & $1.4(1.3-1.6)$ & $1.2(1.0-1.2)$ & 0.122 & \\
\hline $\begin{array}{l}\text { LDL-cholesterol } \\
(\mathrm{mmol} / \mathrm{L})\end{array}$ & $3.3(3.1-3.6)$ & $3.0(2.8-3.2)$ & 0.227 & \\
\hline Glucose (mmol/L) & $5.6(5.1-5.9)$ & $6.5(5.9-7.0)$ & 0.012 & * \\
\hline $\mathrm{HbA} 1 \mathrm{c}(\mathrm{mmol} / \mathrm{mol})$ & $30.0(27.5-31.5)$ & $37.0(34.0-42.0)$ & 0.024 & * \\
\hline ALT $(\mu$ kat $/ \mathrm{L})$ & $0.3(0.3-0.4)$ & $0.5(0.4-0.7)$ & 0.033 & $*$ \\
\hline $\operatorname{ALP}(\mu \mathrm{kat} / \mathrm{L})$ & $1.1(1.0-1.4)$ & $1.3(1.0-1.6)$ & 0.456 & \\
\hline AST ( $\mu$ kat/L) & $0.3(0.3-0.4)$ & $0.4(0.3-0.4)$ & 0.608 & \\
\hline GGT $(\mu \mathrm{kat} / \mathrm{L})$ & $0.3(0.2-0.4)$ & $0.5(0.4-1.0)$ & 0.100 & \\
\hline CRP (mg/L) & $1.0(1.0-1.0)^{\#}$ & $5.5(4.0-7.5)$ & 0.025 & * \\
\hline Total leukocytes $\left(10^{9} / \mathrm{L}\right)$ & $7.4(5.6-7.7)$ & $6.5(6.1-8.2)$ & 0.906 & \\
\hline Platelets $\left(10^{9} / \mathrm{L}\right)$ & $\begin{array}{c}235.0 \\
(196.0-253.5)\end{array}$ & $\begin{array}{c}255.0 \\
(229.5-299.0)\end{array}$ & 0.286 & \\
\hline APTT (sec) & $27.0(25.8-29.5)$ & $29.0(26.0-31.0)$ & 0.913 & \\
\hline $\begin{array}{l}\text { Prothrombin complex } \\
\text { (INR) }\end{array}$ & $1.0(0.9-1.1)$ & $0.9(0.9-1.0)$ & 0.118 & \\
\hline Hemoglobin (g/L) & $\begin{array}{c}140.5 \\
(133.0-143.0)\end{array}$ & $\begin{array}{c}142.0 \\
(129.0-145.5)\end{array}$ & 0.796 & \\
\hline Bilirubin $(\mu \mathrm{mol} / \mathrm{L})$ & $6.1(3.9-9.8)$ & $5.3(4.3-6.4)$ & 0.675 & \\
\hline Creatinine $(\mu \mathrm{mol} / \mathrm{L})$ & $63.0(59.0-65.5)$ & $68.0(61.0-79.5)$ & 0.441 & \\
\hline $\begin{array}{l}\text { Adipocyte size } \\
\text { oWAT }\left(\mu \mathrm{m}^{2}\right)\end{array}$ & 1838 (1658-2004) & $5041(4582-5640)$ & $<0.0001$ & $* * *$ \\
\hline $\begin{array}{l}\text { Adipocyte size } \\
\text { scWAT }\left(\mu \mathrm{m}^{2}\right)\end{array}$ & 3327 (1938-3987) & $5510(4776-6063)$ & 0.0006 & $* * *$ \\
\hline \multicolumn{5}{|c|}{ Medications } \\
\hline Diabetes medications & $0 \%(0 / 8)$ & $13.3 \%(2 / 15)$ & 0.526 & \\
\hline $\begin{array}{l}\text { Anti-hypertensive } \\
\text { medications }\end{array}$ & $0 \%(0 / 8)$ & $46.7 \%(7 / 15)$ & 0.052 & \\
\hline $\begin{array}{l}\text { Lipid lowering } \\
\text { medications }\end{array}$ & $0 \%(0 / 8)$ & $13.3 \%(2 / 15)$ & 0.526 & \\
\hline
\end{tabular}

\# To enable statistical analysis of C-reactive protein (CRP), values which were below the limit of detection $(\mathrm{LOD}=1)$ were replaced with LOD values. $\sigma^{7}$, males; + , females. Abbreviations: BMI, body mass index; BP, blood pressure; HbA1c, glycated hemoglobin; HDL, high-density lipoprotein; LDL, low-density lipoprotein; ALP, alkaline phosphatase; AST, aspartate transaminase; ALT, alanine transaminase; GGT, gamma-glutamyl transferase APTT, activated partial thromboplastin time; INR, international normalized ratio; oWAT, omental white adipose tissue; scWAT, subcutaneous white adipose tissue.

Cardiovascular parameters, namely systolic and diastolic blood pressure, as well as serum triglycerides, were significantly higher in MUO, whereas total cholesterol, HDLcholesterol, and LDL-cholesterol did not differ between the groups. In addition, fasting plasma glucose and $\mathrm{HbA1c}$ were also significantly elevated in MUO. A higher level of serum C-reactive protein (CRP) was detected in the MUO group, possibly indicative of systemic low-grade inflammation. From other blood parameters, such as liver enzymes or hematological parameters, alanine transaminase (ALT) levels were significantly higher 
in MUO compared with MHL. Some of the subjects in the MUO group were taking antihypertensive $(7 / 15)$, diabetes $(2 / 15)$ or lipid lowering $(2 / 15)$ medications, whereas no subjects in the MHL group were treated with these medications. Lastly, adipocyte size was determined in both oWAT and scWAT as median adipocyte area for each subject. As expected, adipocyte size within both fat depots was significantly larger for MUO subjects than for MHL subjects (Table 1).

\subsection{Histological Staining of Collagen in oWAT and scWAT of MHL and MUO Subjects}

To evaluate total ECM deposition, we performed picrosirius red staining of collagen fibrils on oWAT and scWAT sections from MHL and MUO individuals (Figure 1A). Total ECM content, quantified as the total signal intensity from picrosirius red staining divided by the total tissue area, was not different between the oWAT and scWAT depots either when performing paired analysis of all subjects together (mean \pm SEM; oWAT $16.42 \pm 1.43$, scWAT $15.01 \pm 2.41 ; p=0.320$ ) or when comparing oWAT with scWAT for either of the groups separately (Figure 1B). However, when comparing the patient groups with each other, we found total ECM deposition in scWAT to be significantly higher for MHL subjects than MUO individuals (Figure 1B). The difference in oWAT was not significant. The same pattern could be observed for fibrotic bundle staining, as significantly higher staining was detected for MHL when compared with MUO subjects within scWAT, but not oWAT (Figure 1C). In contrast, we observed significantly higher staining of pericellular fibrosis only in oWAT from MHL individuals compared with MUO individuals (Figure 1D). Again, no difference in pericellular fibrosis was identified between the oWAT and scWAT depots for either patient group, or when performing paired comparisons between depots for all subjects together (mean \pm SEM; oWAT $4.18 \pm 0.79$, scWAT $3.03 \pm 0.38 ; p=0.245$ ).

\subsection{Total Collagen Content Assessed by Quantification of Hydroxyproline Content}

To confirm the findings obtained from quantifying picrosirius red staining on histological sections, we carried out a colorimetric assay to determine the total tissue hydroxyproline content in oWAT and scWAT biopsies. Hydroxyproline, a derivate of the amino acid proline, is a major component of the collagen triple helix, and its presence is largely restricted to collagen deposited within the ECM. It is therefore often used as an indicator of total collagen amount. Measuring hydroxyproline showed higher collagen ECM content in the MHL group than the MUO group for both adipose tissue depots, oWAT and scWAT (Figure 1E). In contrast to the picrosirius red staining, paired analysis comparing the hydroxyproline content in oWAT with that of scWAT for all subjects together did result in a significant difference between depots, with higher levels in oWAT (mean \pm SEM; oWAT $0.43 \pm 0.05$, scWAT $0.29 \pm 0.04 ; p<0.001$ ). When comparing either of the patient groups alone, the difference between depots was not significant (Figure 1E). Taken together, the colorimetric hydroxyproline assay confirmed our results from histological sections, showing that the major difference in adipose tissue ECM content was between patient groups, with MHL individuals showing higher levels of tissue collagen than the MUO group. Furthermore, more ECM was detected in oWAT than scWAT when analyzing all subjects together. 
A

OWAT

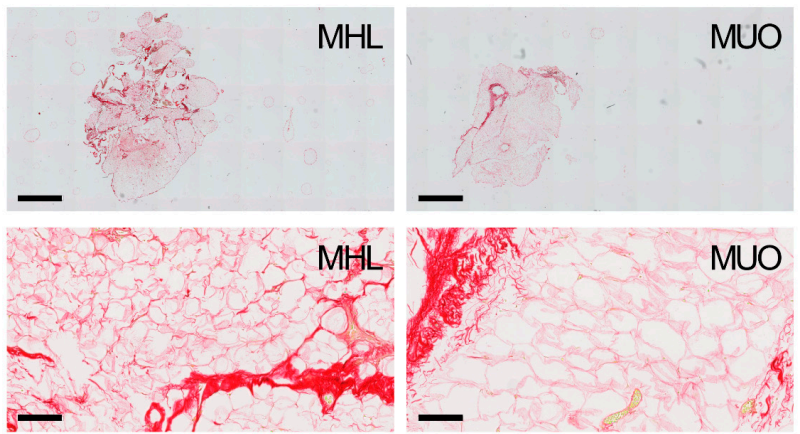

B

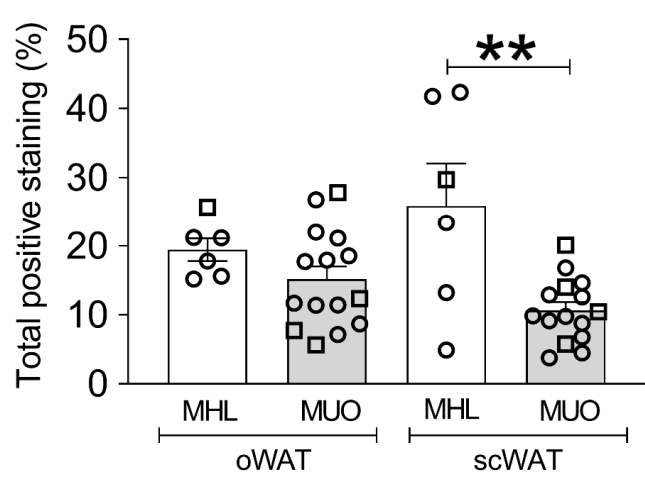

D

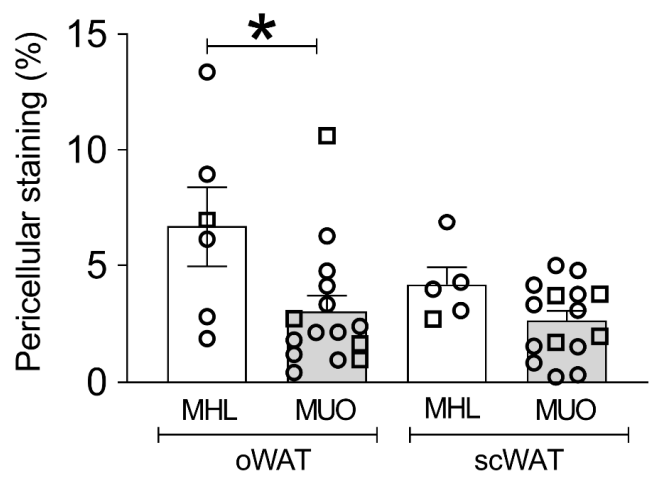

SCWAT

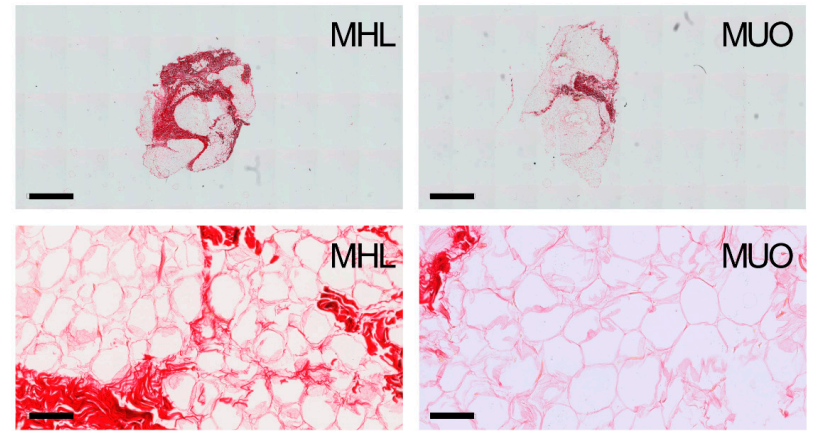

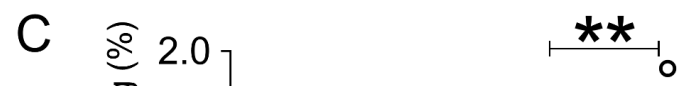

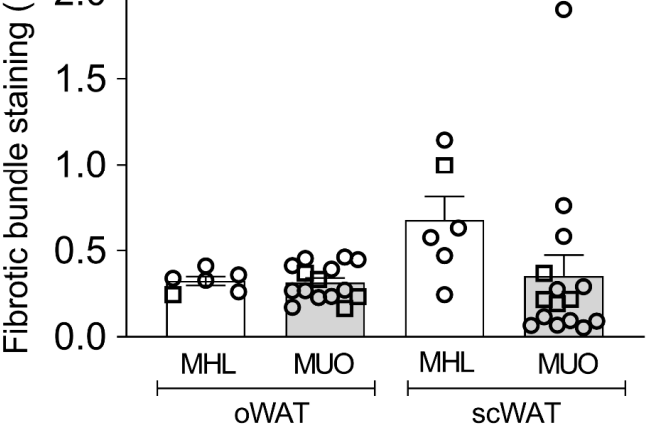

E

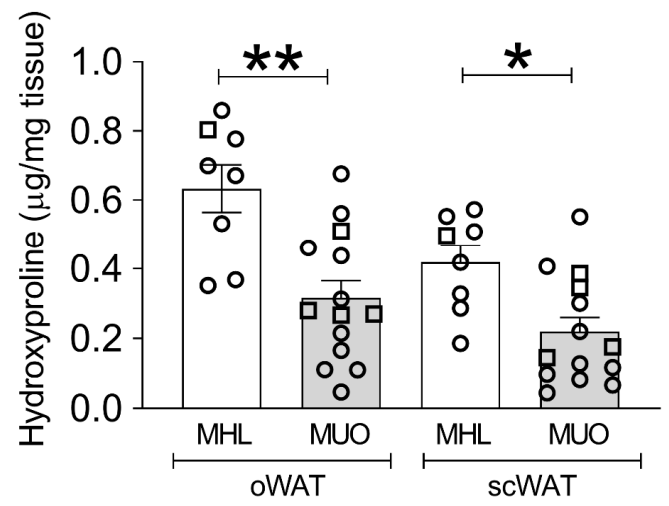

Figure 1. Extracellular matrix (ECM) deposition in omental and subcutaneous white adipose tissue (WAT) of metabolically healthy lean (MHL) and metabolically unhealthy obese (MUO) subjects. (A) Representative images for picrosirius red staining. Scale bar for upper row $=2 \mathrm{~mm}$ and for the second row $=100 \mu \mathrm{m}$. (B-D) Comparison of total ECM staining intensity (B), fibrotic bundle staining (C), and pericellular staining (D) in omental WAT (oWAT) and subcutaneous WAT (scWAT) of $6 \mathrm{MHL}$ and $15 \mathrm{MUO}$ subjects. Data show the amount of stained area as a percentage of the total area and expressed as mean \pm SEM and individual values ( $\bigcirc$ open circles, females; $\square$ open squares, males); (E) Hydroxyproline content in oWAT and scWAT was measured as an indicator of total ECM collagen content in $8 \mathrm{MHL}$ and $14 \mathrm{MUO}$ subjects. Data expressed as mean $\pm \mathrm{SEM}$ and individual values ( $\bigcirc$ open circles, females; $\square$ open squares, males). Statistical analysis was performed using Kruskal-Wallis test followed by Dunn's multiple comparison post-hoc test. ${ }^{*} p \leq 0.05,{ }^{* *} p \leq 0.01$.

\section{4. $m R N A$ Expression of Extracellular Matrix Genes}

To further validate our results and quantify the mRNA expression of genes involved in adipose tissue ECM generation and remodeling in MHL and MUO subjects, we performed a droplet digital polymerase chain reaction (ddPCR) (Figure 2). In line with the results from the collagen staining and the hydroxyproline assays showing more ECM in WAT from lean 
subjects, we found that MHL subjects had a higher transcriptional expression of collagen COL1A1 and the procollagen cleavage enzyme PCOLCE in both fat depots. In contrast, levels of $M M P 9$, involved in ECM degradation, were lower compared with MUO subjects. In addition, MHL subjects displayed higher scWAT expression of smooth muscle actin (encoded by the gene ACTA2), a marker for myofibroblasts, when compared with MUO. Although no significant difference in expression of the hypoxic sensor prolyl 4-hydroxylase (P4HTM) was observed between subject groups, MUO subjects had reduced transcription of the pro-angiogenic factor VEGFA in both depots.
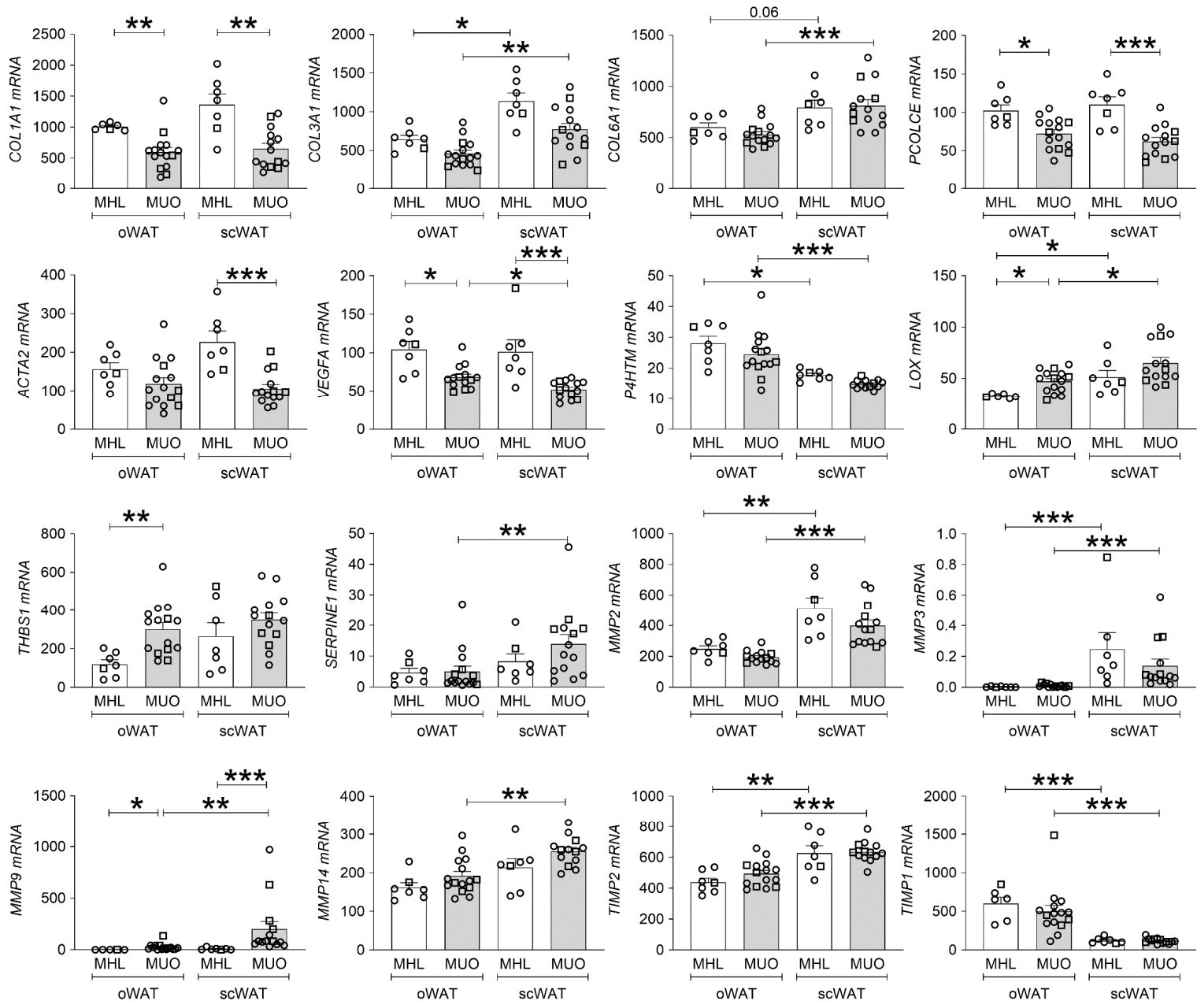

Figure 2. WAT ECM-remodeling gene expression in metabolically healthy lean (MHL) compared with metabolically unhealthy obese (MUO) subjects. mRNA expression data were obtained by ddPCR and calculated as normalized copy number. Data expressed as mean \pm SEM and individual values ( $\bigcirc$ open circles, females; $\square$ open squares, males). Statistical analysis was performed using Kruskal-Wallis test followed by Dunn's multiple comparison post-hoc test. ${ }^{*} p \leq 0.05,{ }^{* *} p \leq 0.01$, *** $p \leq 0.001$.

MUO subjects did however express higher mRNA levels of the pro-fibrotic genes lysyl oxidase (LOX) and thrombospondin 1 (THBS1) in oWAT. LOX is involved in collagen cross-linking and tissue stiffness, while THBS1 is an adhesive glycoprotein that mediates cell-cell and cell-matrix interactions and has been shown to inhibit angiogenesis [22]. These changes are in line with the classical view that obesity leads to fibrosis, especially in the oWAT depot.

The differences between the oWAT and scWAT depots were also assessed. For both subject groups we found higher subcutaneous than omental expression of collagen COL $3 A 1$ and matrix metallopeptidase MMP2, the pro-fibrotic metallopeptidase inhibitor TIMP2 and 
the pro-fibrotic collagen cross-linker LOX. The expression of COL6A1, MMP9, MMP14 and SERPINE1 was only higher in the scWAT from MUO subjects but not in the lean subjects. The expression of metallopeptidase inhibitor TIMP1 and the hypoxic marker P4HTM was lower in scWAT as compared with oWAT for both subject groups. Taken together, the expression analysis confirmed our previous results, suggesting that ECM components were more highly expressed in WAT from MHL subjects than from MUO subjects. Moreover, the expression analysis showed scWAT to be more likely to express components involved in ECM remodeling than oWAT, suggesting that high expression of ECM remodeling enzymes could have beneficial effects on tissue function.

\subsection{Correlation between Collagen Content, Clinical Parameters and mRNA Expression}

To further evaluate the relationship between ECM markers and metabolic phenotype, we performed correlation analysis between clinical parameters and total hydroxyproline content in oWAT and scWAT, respectively. Hydroxyproline content correlated significantly to the total picrosirius red staining intensity (Spearman's $r_{s}=0.39, p=0.014$ ). Body weight and BMI were negatively correlated with WAT hydroxyproline content in both fat depots, and waist circumference negatively correlated with hydroxyproline content in oWAT. Spearman's correlation coefficients and respective $p$ values are shown in Table 2. In line with this, a significant negative correlation was determined between hydroxyproline content and adipocyte size in oWAT, whereas the correlation did not reach statistical significance $(p=0.092)$ in scWAT. In addition, diastolic blood pressure displayed a negative correlation with ECM content in both oWAT and scWAT depots. Finally, HDL-cholesterol levels in serum were positively correlated with hydroxyproline content in oWAT, but not scWAT.

Table 2. Correlation analysis between clinical parameters and hydroxyproline content $(\mu \mathrm{g} / \mathrm{mg}$ tissue) in oWAT and scWAT, respectively. $r_{s}$, Spearman's rank correlation coefficient; $p$ value, the statistical significance of Spearman's correlation test. Blue and red colors indicate a significant negative and positive correlation, respectively.

\begin{tabular}{ccccc}
\hline & \multicolumn{2}{c}{ oWAT ECM } & \multicolumn{2}{c}{ scWAT ECM } \\
\cline { 2 - 5 } & $\mathbf{r}_{\mathbf{s}}$ & $\boldsymbol{p}$ Value & $\mathbf{r}_{\mathbf{s}}$ & $\boldsymbol{p}$ Value \\
\hline Age & 0.238 & 0.286 & 0.055 & 0.806 \\
Body weight & -0.585 & 0.004 & -0.465 & 0.029 \\
BMI & -0.621 & 0.003 & -0.461 & 0.032 \\
Waist circumference & -0.569 & 0.006 & -0.419 & 0.052 \\
\hline BP systolic & -0.414 & 0.055 & -0.252 & 0.258 \\
BP diastolic & -0.792 & $<0.001$ & -0.504 & 0.017 \\
Heart Rate & -0.311 & 0.183 & -0.308 & 0.186 \\
\hline Glucose & -0.176 & 0.471 & -0.271 & 0.262 \\
HbA1C & -0.128 & 0.625 & 0.169 & 0.518 \\
Cholesterol & 0.365 & 0.150 & 0.180 & 0.490 \\
HDL-cholesterol & 0.481 & 0.050 & 0.163 & 0.532 \\
LDL-cholesterol & 0.333 & 0.192 & 0.203 & 0.435 \\
Triglycerides & -0.269 & 0.297 & -0.025 & 0.926 \\
\hline CRP & -0.269 & 0.296 & -0.090 & 0.732 \\
ALP & -0.150 & 0.506 & -0.044 & 0.845 \\
AST & 0.018 & 0.936 & 0.164 & 0.465 \\
ALT & -0.293 & 0.185 & -0.126 & 0.576 \\
GGT & -0.160 & 0.540 & -0.053 & 0.840 \\
\hline Total leukocytes & -0.206 & 0.427 & 0.026 & 0.922 \\
Platelets & -0.372 & 0.142 & -0.343 & 0.177 \\
APTT & -0.061 & 0.800 & -0.211 & 0.372 \\
\hline & & & &
\end{tabular}


Table 2. Cont.

\begin{tabular}{ccccc}
\hline & \multicolumn{2}{c}{ oWAT ECM } & \multicolumn{2}{c}{ scWAT ECM } \\
\cline { 2 - 5 } & $\mathbf{r}_{\mathbf{s}}$ & $\boldsymbol{p}$ Value & $\mathbf{r}_{\mathbf{s}}$ & $\boldsymbol{p}$ Value \\
\hline Prothrombin complex & 0.164 & 0.489 & 0.156 & 0.512 \\
Hemoglobin & -0.058 & 0.797 & -0.226 & 0.312 \\
Bilirubin & 0.011 & 0.960 & -0.291 & 0.189 \\
Creatinine & -0.023 & 0.929 & -0.177 & 0.497 \\
\hline Adipocyte size (median) & -0.629 & 0.004 & -0.388 & 0.092 \\
\hline
\end{tabular}

Abbreviations: BMI, body mass index; BP, blood pressure; HbA1c, glycated hemoglobin; HDL, high-density lipoprotein cholesterol; LDL, low-density lipoprotein cholesterol; ALP, alkaline phosphatase; AST, aspartate transaminase; ALT, alanine transaminase; GGT, gamma-glutamyl transferase; APTT, activated partial thromboplastin time.

\section{Discussion}

In order to design better therapies against obesity-induced metabolic diseases it is important to understand the pathological changes related to obesity, specifically in human adipose tissue. Here, we used a combination of three established techniques (picrosirius red staining, a colorimetric hydroxyproline assay and ddPCR measurement of gene expression) to evaluate ECM deposition in the two major human fat depots, oWAT and scWAT, from MHL and MUO subjects. The subject groups were selected to distinguish between the physiological WAT morphology seen in the MHL group and pathological WAT remodeling associated with obesity and metabolic disease in the MUO group.

\subsection{ECM Specific Differences between Metabolic Groups}

Our results indicate that the MHL group is characterized by robust deposition and expression of ECM components compared with the group of MUO individuals. This finding may appear surprising, as many reports show that obesity is associated with higher WAT fibrosis $[12,13,23,24]$, especially in the oWAT depot. Seminal papers published by the Clément group repeatedly demonstrate that fibrosis was significantly higher in obesity in both human oWAT and scWAT $[12,13]$. However, it is noteworthy that the role of adipose tissue fibrosis in humans has been more debated in recent years [25].

We argue that confounding factors may be important when reflecting upon the differences in results. Previous studies did not separate metabolically healthy and unhealthy obese subjects [13], which may be an important variable to consider. We chose to specifically compare healthy, lean individuals to obese subjects that presented with a metabolically unhealthy phenotype.

Elegant studies by Scherer and colleagues have shown that WAT hypoxia is a known contributor to obesity-related WAT fibrosis in mice [26]. We did not observe higher gene expression of the oxygen sensor P4HTM, indicating unaltered HIF1 $\alpha$ signaling. However, expression levels of the pro-angiogenic factor VEGFA was reduced in MUO. This disconnect between oxygen and VEGF-A signaling pathways has been described before, where HIF1 $\alpha$ expression in WAT failed to induce the known target gene VEGFA [26]. VEGF-A has also been linked to tissue fibrosis [8,27], and thus the lower gene expression of this gene supports our findings of reduced fibrosis in the MUO group. These data suggest a more complex crosstalk between oxygen sensing pathways, such as HIF $1 \alpha$, pro-angiogenic signaling and WAT fibrosis.

Discrepancies in the field might also be due to different studies quantifying, at least in part, different features: some focusing only on pathological fibrosis, whereas others, such as ours, measure total ECM deposition in the tissue. In general, we find that studies measuring the accumulation of specific, pro-fibrotic collagens, including collagen IV, V and VI, seem to more often report a positive correlation between BMI and WAT fibrosis, underlining the pathologic role of their accumulation [13,16,28-30]. Several papers, but far from all, which quantify total WAT ECM either by staining or colorimetric assays have found a negative association between total ECM deposition and subject BMI and/or the metabolic health, 
in line with our results $[18,20,31]$. There might also exist other qualitative differences in ECM deposition besides collagen type during WAT expansion. Whereas Spencer et al. found significantly higher collagen V deposition in obese scWAT biopsies compared with lean, elastin in the same obese subjects was found to be lower [16]. Other studies report similar findings to our data. Henegar et al. reported less COL1A1 expression in the obese population and more expression of the pro-fibrotic collagen cross-linker LOX [13]. This manuscript also reported higher expression of the more pro-fibrotic collagens COL4A1, COL5A2 and COL12A1 [13].

Similar to our observations, a study by Divoux et al. identified a positive correlation between ECM deposition in oWAT and HDL-cholesterol levels, and a negative association between ECM staining and adipocyte size in the same depot, suggesting that adipocyte hypertrophy is associated with less ECM deposition. The authors also speculate that reduced ECM in obesity potentially increases the capacity for the cells to enlarge [12]. However, this opposes another hypothesis, whereby higher ECM stiffness in obesity (despite reduced expression of ECM components) mechanically stabilizes the adipocytes but prevents the cells from expanding, contributing to developing a metabolically unhealthy phenotype [32]. Our results demonstrate higher LOX activity in obesity, suggesting enhanced ECM crosslinking and higher adipocyte tissue stiffness. Enhanced LOX activity has been shown to increase adipose tissue stiffness in $o b / o b$ mice and obese individuals, which was reversed after weight loss surgery [33].

Our results further highlight that robust expression of components involved in ECM remodeling (such as collagens, PCOLCE and ACTA2) could be a sign of WAT health. We speculate that robust expression of ECM remodeling enzymes might enable WAT to dynamically adapt to various changes in triglyceride accumulation and energy levels, whereas elevated expression of LOX and THBS1 contributes to tissue stiffness and accumulation of more fibrotic ECM components.

Summarized, these results highlight the need for a redefinition of WAT fibrosis, starting with differentiating between total ECM accumulation, which sometimes might have a physiological role, and pathological changes to the WAT microenvironment. In contrast to fibrotic staining techniques, higher WAT stiffness, although difficult to measure in experimental laboratories, seems to most often correlate positively with parameters of metabolic disease, and could be the most rigid feature of unhealthy WAT expansion that should be measured more often $[18,31,33]$. More techniques to experimentally assess WAT ECM stiffness in adipose tissue fibrosis are therefore warranted. Meta-analyses will also aid to identify specific ECM components involved in physiological and pathological ECM remodeling. We speculate that in the future, detailed investigations of WAT ECM could potentially allow for the development of diagnostic methods to identify these changes in early stages of obesity and, from there, develop interventions that prevent the occurrence of inflammatory processes or oxidative damage associated with obesity and related complications such as type 2 diabetes.

\subsection{Differences between oWAT and scWAT}

Another unexpected result was that we did not find any significant difference in picrosirius red staining when comparing paired oWAT versus scWAT tissue biopsies. This result is somewhat reflected in our expression analyses, where we could only identify higher expression of COL3A1 in scWAT compared to oWAT, and an higher COL6A1 expression only in scWAT of MUO individuals. However, we consistently found higher scWAT expression of several genes coding for proteins involved in ECM remodeling and cross-linking compared with oWAT, including MMP2 or TIMP2. These changes indicate that scWAT appears to be more dynamically regulated compared to oWAT. In line with this observation, oWAT showed almost no expression of matrix metalloproteinases MMP3 and MMP9, while displaying high levels of the MMP inhibitor TIMP1. Looking at specific substrates for MMP3 and MMP9 revealed that both enzymes target several collagen classes, including collagens III and IV [34]. Collagen III forms the structural framework in adipose 
tissue, while collagen IV is associated with the formation of the basal lamina, providing structural support for the tissue [35]. Low expression of these MMPs, with higher expression of TIMP1, which inhibits nearly all matrix metalloproteinases [36], further supports lower adaptability of the oWAT fat depot compared with scWAT.

\subsection{Summary}

We propose that healthy WAT is characterized by robust ECM deposition and turnover. Conversely, low total ECM content with higher rigidity might be a characteristic of obese, unhealthy WAT in humans (Figure 3). Our results align with the hypothesis that in the lean state, the ECM of WAT acts favorably to allow dynamic expansion of adipocytes, despite higher collagen levels. A reduction in ECM remodeling by higher collagen cross-linkage by LOX and enhanced cell-cell and cell-matrix interactions through THBS1, lead to developing WAT dysfunction that characterizes unhealthy obesity.

\section{Healthy adipose tissue in lean subjects}

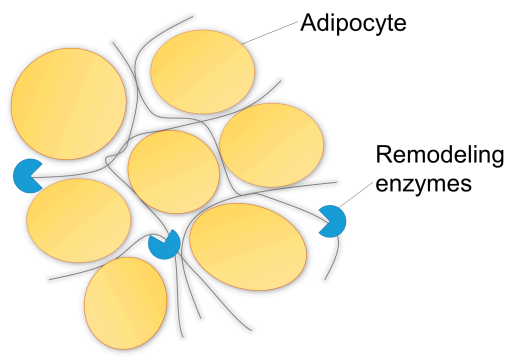

Healthy adipose tissue in lean subjects readily adaptable to remodel ECM.

ECM
$\begin{gathered}\text { Remodeling } \\ \text { enzymes }\end{gathered}$

\section{Obese adipose tissue}

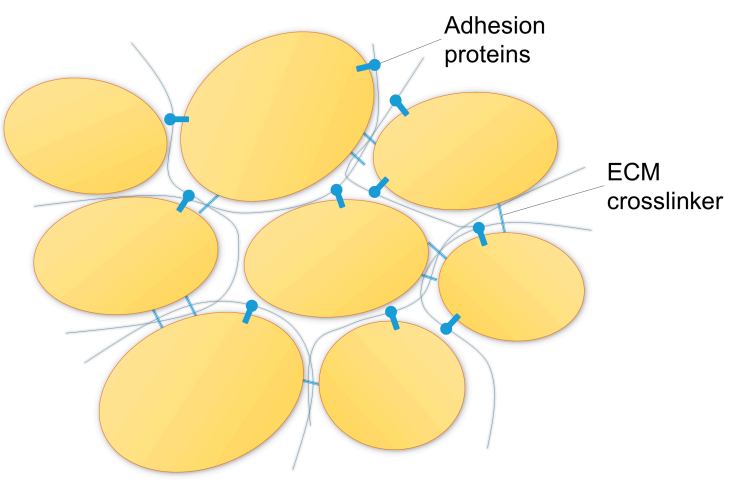

Obese adipose tissue more rigid and less dynamic.

$\begin{array}{cc}\text { ECM } & \downarrow \\ \text { Crosslinking } \\ \text { enzymes }\end{array}$

Figure 3. Summarized main findings highlighting the differences between healthy adipose tissue in lean subjects and adipose tissue in metabolically unhealthy obese individuals. ECM, extracellular matrix.

Taken together, our results measuring the ECM status in MHL and MUO subjects underline the marked difference between physiological ECM accumulation and pathological fibrosis. Data from our experiments also highlight the importance of understanding what exactly the measured parameters are quantifying. Part of the discrepancy between the results reported here and the current view of the field might stem from differences in recruited patients, techniques used, and genes measured. However, our report is by no mean the first to find a negative association between WAT collagen content and patient BMI, and highlights the need for further studies and in-depth discussions. Our data also suggest that quantifying WAT stiffness and remodeling might be better measurements of the pathological state associated with obesity than picrosirius red staining alone.

\subsection{Study Limitations}

Several limitations may be taken into consideration when interpreting our study results. First, the number of participants is limited $(n=8$ and 15 in MHL and MUO groups, respectively), although the groups were carefully characterized and multiple methods applied to analyze the ECM deposition. Although both males and females were included in the study, the sexes were not equally distributed as females were overrepresented in both 
groups. Hence, our analyses do not allow for a sex-dependent statistical evaluation. This is an important consideration, as reports show that males and females have differences in adipose tissue physiology and pathophysiology [37]. However, when presenting our data as individual data points where the sex of each individual is shown, we could not observe any apparent skewness of the data due to sex. Adipose tissue biology is also affected by hormones such as estrogen [38]. None of the participants included in these analyses received hormonal or contraceptive treatment, however, we did not collect the information about the menopausal status of the participants.

The extreme differences in fat mass and adipocyte size between lean and obese subjects make it difficult to directly compare extracellular content with suitable normalization strategy. We normalized values according to widely used strategies in the field (e.g., picrosirius red staining normalized to tissue area, ECM content in relation to tissue weight, and RNA expression in relation to housekeeping genes that show stable expression in human adipose tissue) $[12,13,20,39,40]$. This makes our results comparable with most published studies in the field. However, further studies that use larger patient cohorts and characterize the relative influence of ECM quantification in relation to various normalization strategies (including that of adipocyte cell size) would represent a valuable addition to the field.

Another important consideration is that gastric bypass patients underwent a presurgical diet prior to tissue collection and we cannot exclude the possibility that this may have an affect (e.g., in our transcriptome analyses). However, previous studies by Clément and colleagues have shown that short-term caloric restriction increases adipose tissue ECM deposition in obese gastric bypass patients [41]. Thus, the trend shown in our study whereby lean subjects have more ECM as compared with obese subjects would likely be even more pronounced if analyzing adipose tissue obtained prior to the pre-surgical diet.

Finally, another limitation is that since we did not investigate protein expression in this study, we do not know if the statistically significant differences in gene expression with various size effects translate into protein expression and protein function. Although the effect size is rather small in the expression of some genes, it can still translate into significant functional differences, especially in the case of enzymes.

\section{Materials and Methods}

\subsection{Study Participants}

Obese subjects [body mass index (BMI) median and interquartile range (IQR) 43.2 $(39.6-44.9) \mathrm{kg} / \mathrm{m}^{2}$, aged $47.0(35.0-54.0)$ years] were recruited from a group of patients scheduled to undergo laparoscopic gastric bypass surgery and lean controls [BMI, median and IQR $22.2(21.7-23.2) \mathrm{kg} / \mathrm{m}^{2}$, aged 40.0 (33.8-47.2) years] were recruited from patients scheduled for non-acute cholecystectomy due to uncomplicated bile stone disease. The volunteer patients were subsequently characterized as metabolically healthy or unhealthy, based on the International Diabetes Federation (IDF) criteria of the metabolic syndrome [21]. Thus, obese subjects included in the study displayed three or more metabolic syndrome components in order to be characterized as MUO and match the recruitment criteria.

Importantly, fat biopsies collected during gastric bypass and cholecystectomies were taken from the same anatomical subregions, which is pivotal when comparing patients undergoing different types of surgeries.

MUO subjects scheduled for gastric bypass underwent short-term low-calorie diet restriction three weeks before surgery. Therefore, the anthropometric measurements and blood collection were performed before the start of the pre-surgical dieting, to avoid the effect of short-term calorie restriction. For MHL subjects, all samples were collected on the day of surgery. Subjects were excluded if they were taking anti-inflammatory and/or immunosuppressive drugs, currently smoked, or were diagnosed with significant gastrointestinal disease or inflammatory bowel disease. 
The study was approved by the Swedish Ethical Review Authority \#682-14 (ClinicalTrials.gov NCT02322073). All procedures were carried out in accordance with the Helsinki Declaration and written informed consent was obtained from all participants.

\subsection{Plasma and Serum Preparation and Biochemical Laboratory Analyses}

Certified nurses collected venous blood into $\mathrm{K}_{2}$ EDTA tubes (\#456243, Greiner Bio-One, Kremsmünster, Austria) for anticoagulated blood or plasma preparation and Z Serum Separator Clot Activator tubes (\#454420, Greiner Bio-One) for serum, as previously described [42]. Hematological and biochemical parameters of blood, plasma or serum were assessed by an accredited hospital laboratory (Sahlgrenska University Hospital, Gothenburg, Sweden).

\subsection{Adipose Tissue Samples}

Paired oWAT and scWAT samples were obtained from MHL and MUO subjects from the respective sites during surgery and immediately processed further. Tissue samples for RNA analysis and the hydroxyproline assay were snap frozen in liquid nitrogen and stored at $-80{ }^{\circ} \mathrm{C}$ before analysis. Moreover, samples were also fixed in $4 \%$ formaldehyde and processed for paraffin embedding for immunohistochemistry.

\subsection{Immunohistochemistry Analysis}

Paraffin embedded formaldehyde fixed oWAT and scWAT biopsies were sectioned (5 $\mathrm{mm}$ /section) and stained with Picrosirius Red (Histocenter, Gothenburg, Sweden; accredited by Swedac 17,025:2005). Images were acquired at 20× magnification on the Metafer Slide Scanning Platform (Altlussheim, Germany) and image analysis was carried out using Aperio ImageScope software (version. 12.4.0.7018). Total fibrosis was quantified as percentage of picrosirius red stained tissue area divided by the total tissue area, as described by Henegar et al. [13]. Similarly, pericellular fibrosis was determined by examining the picrosirius red staining in 2-10 randomly chosen $10 \times$ fields in parts of the tissue free of fibrotic bundles and expressed as the percentage of the sum of stained areas divided by the total sum of field surfaces. The semi-automatic image analysis using a manually optimized algorithm classified positively stained pixels into strong positive, positive and weak positive. Fibrotic bundle staining was determined by taking the ratio of strong positively stained pixels and dividing them by the positively + weakly positive stained pixels.

To estimate adipocyte size, intact adipocytes were manually circled and the median adipocyte area was calculated using the Aperio ImageScope analysis software (version. 12.4.0.7018).

\subsection{Hydroxyproline Assay}

Hydroxyproline content was measured using a hydroxyproline assay kit (\#MAK008, Sigma-Aldrich, St. Louis, MO, USA). Briefly, $20 \mathrm{mg}$ frozen adipose tissue pieces were weighed and homogenized in $200 \mu \mathrm{L}$ water. $100 \mu \mathrm{L}$ of the lysate was then incubated with $100 \mu \mathrm{L} 12 \mathrm{M} \mathrm{HCl}$ at $120^{\circ} \mathrm{C}$ for $3 \mathrm{~h}$. $10 \mu \mathrm{L}$ of the hydrolyzed samples were dried before incubation with chloramine-T and p-dimethyl-amino-benzaldehyde at $60{ }^{\circ} \mathrm{C}$ for $90 \mathrm{~min}$. Absorbance was measured at $560 \mathrm{~nm}$, and the concentrations of the samples were determined using a standard curve generated with hydroxyproline provided in the kit.

\subsection{RNA Isolation}

Frozen adipose tissue (approximately $100 \mathrm{mg}$ ) was homogenized in $1 \mathrm{~mL}$ of Trizol reagent (\#15596018, Invitrogen, Waltham, MA, USA) with $5 \mathrm{~mm}$ Stainless Steel Bead (\#69989, Qiagen, Hilden, Germany) using TissueLyser (Qiagen). After 10 min incubation, the homogenate was centrifuged $(12,000 \times g)$. According to the manufacturer's instructions, the top lipid layer was discarded and $0.8 \mathrm{~mL}$ of supernatant was used for subsequent chloroform extraction until an upper water phase with RNA was formed and transferred to the new tube. An equal volume of $70 \%$ ethanol was added and mixed thoroughly. The 
mixture was transferred to RNeasy Mini Kit column (\#74106, Qiagen), and total RNA was isolated according to the manufacturer's instructions. RNA was eluted in $30 \mu \mathrm{L}$ of PCR grade water. Concentration and purity of RNA were assessed using NanoDrop 2000 (ThermoFisher Scientific, Waltham, MA, USA) and RNA integrity was analyzed using RNA Pico Chips (\#5067-1513, Agilent Technologies, Waldbronn, Germany) and the Agilent 2100 Bioanalyzer (Agilent Technologies).

\subsection{Reverse Transcription and Droplet Digital Polymerase Chain Reaction}

$0.5 \mu \mathrm{g}$ total RNA was treated with DNase I (\#18068015, Invitrogen) and first strand cDNA was synthesized in $20 \mu \mathrm{L}$ reaction volume using random primers (\#48190011, Invitrogen), dNTP mix, DTT, RNase inhibitor (\#10777019, Invitrogen), and SuperScript IV Reverse Transcriptase (\#18090050, Invitrogen). Gene expression was evaluated by droplet digital PCR (ddPCR) using QX200 AutoDG Droplet Digital PCR System (\#1864100, BioRad, Hercules, CA, USA). $22 \mu \mathrm{L}$ of PCR reaction mix comprised of ddPCR Supermix for Probes without dUTP (\#1863023, Bio-Rad), specific target (FAM-labeled) and reference (HEX-labeled) PrimePCR ddPCR Expression Probe Assay (Table S1) and $2 \mu \mathrm{L}$ of appropriately diluted template cDNA $(3 \times$, or $30 \times$, respectively). Single droplets were generated in DG32 ${ }^{\mathrm{TM}}$ Automated Droplet Generator Cartridges (\#1864108, Bio-Rad) using Automated Droplet Generator (\#1864101, Bio-Rad) and Automated Droplet Generation Oil for Probes (\#1864110, Bio-Rad). PCR reaction was run in C1000 Touch Thermal Cycler (Bio-Rad) according to the manufacturer's instructions $\left(95^{\circ} \mathrm{C}\right.$ for $10 \mathrm{~min}$ for enzyme activation, 40 cycles of denaturation at $94{ }^{\circ} \mathrm{C}$ for $30 \mathrm{~s}$ followed by annealing and extension at $60{ }^{\circ} \mathrm{C}$ for $1 \mathrm{~min}, 98^{\circ} \mathrm{C}$ for $10 \mathrm{~min}$ for enzyme denaturation, $4^{\circ} \mathrm{C}$ overnight). Droplet reading was performed using the QX200 Droplet Reader (\#1864003, Bio-Rad), and data was analyzed using QuantaSoft software (ver. 1.7.4, Bio-Rad). The same manual threshold settings were applied for each specific target assay across all samples. The absolute number of mRNA copies per $\mu \mathrm{L}$ was assessed, and the value per $1 \mathrm{ng}$ of input total RNA was calculated for each target and reference gene. For gene expression normalization, two reference internal control genes (LRP10, RPLP0) stably expressed in human adipose tissue [39] were selected, and the normalization factor was calculated as a geometric mean of their values normalized to maximal RNA copy value of each gene [43]. Normalized copy number was calculated as absolute mRNA copy number per $1 \mathrm{ng}$ RNA divided by the normalization factor. When the group consisted of $n \geq 6$, Grubb's test was used on $\log _{2}$ values to identify outliers.

\subsection{Statistical Analyses}

Spearman's correlation tests were used to calculate the Spearman's rank correlation coefficient $\left(\mathrm{r}_{\mathrm{s}}\right)$ and $p$ values between clinical parameters and the hydroxyproline content or mRNA expression in oWAT and scWAT, respectively. MHL and MUO groups were pooled to allow a sufficient number for correlation analysis. There were missing values for some clinical parameters, and these were excluded as only paired values were included in the correlation analysis for each specific parameter.

The differences in clinical parameters or adipocyte size between MHL and MUO were assessed using the Mann-Whitney $U$ test. Fibrosis quantification, hydroxyproline content and mRNA expression were analyzed using the Kruskal-Wallis test with Dunn's post-hoc pairwise test. To assess difference between oWAT and scWAT in total fibrosis, pericellular fibrosis and hydroxyproline content, the Wilcoxon signed-rank test was used for matched samples. $p$ values $\leq 0.05$ were considered statistically significant. Analyses were performed in GraphPad Prism (ver. 8.4.3) or R environment (R ver. 4.1.0).

\section{Conclusions}

We found robust ECM deposition in MHL subjects with a higher expression of several components implicated in ECM production and remodeling. MUO individuals displayed lower expression and deposition of ECM components but showed higher expression of genes coding cross-linking and adhesion proteins. We conclude that high ECM deposition 
and remodeling might be a key signature of healthy adipose tissue. Our study also suggests that subcutaneous fat is generally more adaptable than omental adipose tissue, owing to the higher expression of proteins involved in ECM remodeling. Further studies are needed to understand what differentiates physiological ECM deposition from pathological fibrosis, and what methods are most suited to measure the two conditions in human fat.

Supplementary Materials: The following supporting information can be downloaded at: https: //www.mdpi.com/article/10.3390/ijms23010520/s1.

Author Contributions: Conceptualization, M.S., M.R.R., C.E.H. and E.B.; methodology, M.S., M.R.R., M.C., V.W., C.E.H. and E.B.; formal analysis, M.S., M.R.R., C.E.H. and E.B.; investigation, M.S., M.R.R., M.C., C.B., V.W. and E.B.; resources, V.W. and E.B.; data curation, M.S. and M.R.R.; writing—original draft preparation, M.S., M.R.R., C.E.H. and E.B.; writing-review and editing, M.S., M.R.R., V.W., C.E.H. and E.B.; visualization, M.S. and M.R.R.; supervision, E.B.; project administration, C.B. and E.B.; funding acquisition, E.B. All authors have read and agreed to the published version of the manuscript.

Funding: The Börgeson laboratory is supported by the Wallenberg Centre for Molecular \& Translational Medicine at the University of Gothenburg and Knut \& Alice Wallenberg Foundation, the Swedish Research Council (\#2016/82), SSMF (\#S150086), and an ERC StG (\#804418). C.E.H is supported by the Swedish Research Council (\#2019-02046), Åke Wibergs stiftelse (M19-0250 \& M20-0122) and Karolinska Institutet (\#2-1062/2018).

Institutional Review Board Statement: The study was approved by the Swedish Ethical Review Authority \#682-14 (ClinicalTrials.gov NCT02322073). All procedures were carried out under the guidelines of the Declaration of Helsinki, and written informed consent was obtained from all participants.

Informed Consent Statement: Informed consent was obtained from all subjects involved in the study.

Data Availability Statement: Data are contained within the article or Supplementary Material.

Acknowledgments: We gratefully acknowledge the contribution of Catherine Åhlund, who with C.B. was responsible for organizing the collection of clinical material. We also acknowledge the kind assistance of coordinators and nurses at the Angered Hospital and the Sahlgrenska Hospital, and the assistance of collaborating surgeons, specifically Leif Nelvin, Per Björklund, Malin Ragnmark Ek and Torsten Olbers.

Conflicts of Interest: The authors declare no conflict of interest.

\section{References}

1. Chait, A.; den Hartigh, L.J. Adipose tissue distribution, inflammation and its metabolic consequences, including diabetes and cardiovascular disease. Front. Cardiovasc. Med. 2020, 7, 22. [CrossRef] [PubMed]

2. Lee, M.J.; Wu, Y.; Fried, S.K. Adipose tissue heterogeneity: Implication of depot differences in adipose tissue for obesity complications. Mol. Asp. Med. 2013, 34, 1-11. [CrossRef] [PubMed]

3. Ibrahim, M.M. Subcutaneous and visceral adipose tissue: Structural and functional differences. Obes. Rev. 2010, 11, 11-18. [CrossRef]

4. Wajchenberg, B.L. Subcutaneous and visceral adipose tissue: Their relation to the metabolic syndrome. Endocr. Rev. 2000, 21, 697-738. [CrossRef] [PubMed]

5. Lotta, L.A.; Gulati, P.; Day, F.R.; Payne, F.; Ongen, H.; van de Bunt, M.; Gaulton, K.J.; Eicher, J.D.; Sharp, S.J.; Luan, J.; et al. Integrative genomic analysis implicates limited peripheral adipose storage capacity in the pathogenesis of human insulin resistance. Nat. Genet. 2017, 49, 17-26. [CrossRef]

6. Williams, A.S.; Kang, L.; Wasserman, D.H. The extracellular matrix and insulin resistance. Trends Endocrinol. Metab. 2015, 26, 357-366. [CrossRef]

7. Ruiz-Ojeda, F.J.; Mendez-Gutierrez, A.; Aguilera, C.M.; Plaza-Diaz, J. Extracellular matrix remodeling of adipose tissue in obesity and metabolic diseases. Int. J. Mol. Sci. 2019, 20, 4888. [CrossRef]

8. Hammarstedt, A.; Gogg, S.; Hedjazifar, S.; Nerstedt, A.; Smith, U. Impaired adipogenesis and dysfunctional adipose tissue in human hypertrophic obesity. Physiol. Rev. 2018, 98, 1911-1941. [CrossRef]

9. Sun, K.; Kusminski, C.M.; Scherer, P.E. Adipose tissue remodeling and obesity. J. Clin. Investig. 2011, 121, 2094-2101. [CrossRef]

10. Choe, S.S.; Huh, J.Y.; Hwang, I.J.; Kim, J.I.; Kim, J.B. Adipose tissue remodeling: Its role in energy metabolism and metabolic disorders. Front. Endocrinol. 2016, 7, 30. [CrossRef] 
11. Crewe, C.; An, Y.A.; Scherer, P.E. The ominous triad of adipose tissue dysfunction: Inflammation, fibrosis, and impaired angiogenesis. J. Clin. Investig. 2017, 127, 74-82. [CrossRef]

12. Divoux, A.; Tordjman, J.; Lacasa, D.; Veyrie, N.; Hugol, D.; Aissat, A.; Basdevant, A.; Guerre-Millo, M.; Poitou, C.; Zucker, J.D.; et al. Fibrosis in human adipose tissue: Composition, distribution, and link with lipid metabolism and fat mass loss. Diabetes 2010, 59, 2817-2825. [CrossRef]

13. Henegar, C.; Tordjman, J.; Achard, V.; Lacasa, D.; Cremer, I.; Guerre-Millo, M.; Poitou, C.; Basdevant, A.; Stich, V.; Viguerie, N.; et al. Adipose tissue transcriptomic signature highlights the pathological relevance of extracellular matrix in human obesity. Genome Biol. 2008, 9, R14. [CrossRef] [PubMed]

14. Sun, K.; Tordjman, J.; Clement, K.; Scherer, P.E. Fibrosis and adipose tissue dysfunction. Cell Metab. 2013, 18, 470-477. [CrossRef] [PubMed]

15. Khan, T.; Muise, E.S.; Iyengar, P.; Wang, Z.V.; Chandalia, M.; Abate, N.; Zhang, B.B.; Bonaldo, P.; Chua, S.; Scherer, P.E. Metabolic dysregulation and adipose tissue fibrosis: Role of collagen VI. Mol. Cell Biol. 2009, 29, 1575-1591. [CrossRef] [PubMed]

16. Spencer, M.; Unal, R.; Zhu, B.; Rasouli, N.; McGehee, R.E., Jr.; Peterson, C.A.; Kern, P.A. Adipose tissue extracellular matrix and vascular abnormalities in obesity and insulin resistance. J. Clin. Endocrinol. Metab. 2011, 96, E1990-E1998. [CrossRef] [PubMed]

17. Michaud, A.; Tordjman, J.; Pelletier, M.; Liu, Y.; Laforest, S.; Noel, S.; Le Naour, G.; Bouchard, C.; Clement, K.; Tchernof, A. Relevance of omental pericellular adipose tissue collagen in the pathophysiology of human abdominal obesity and related cardiometabolic risk. Int. J. Obes. 2016, 40, 1823-1831. [CrossRef] [PubMed]

18. Lackey, D.E.; Burk, D.H.; Ali, M.R.; Mostaedi, R.; Smith, W.H.; Park, J.; Scherer, P.E.; Seay, S.A.; McCoin, C.S.; Bonaldo, P.; et al. Contributions of adipose tissue architectural and tensile properties toward defining healthy and unhealthy obesity. Am. J. Physiol. Endocrinol. Metab. 2014, 306, E233-E246. [CrossRef]

19. McCulloch, L.J.; Rawling, T.J.; Sjoholm, K.; Franck, N.; Dankel, S.N.; Price, E.J.; Knight, B.; Liversedge, N.H.; Mellgren, G.; Nystrom, F.; et al. COL6A3 is regulated by leptin in human adipose tissue and reduced in obesity. Endocrinology 2015, 156, 134-146. [CrossRef]

20. Muir, L.A.; Neeley, C.K.; Meyer, K.A.; Baker, N.A.; Brosius, A.M.; Washabaugh, A.R.; Varban, O.A.; Finks, J.F.; Zamarron, B.F.; Flesher, C.G.; et al. Adipose tissue fibrosis, hypertrophy, and hyperplasia: Correlations with diabetes in human obesity. Obesity 2016, 24, 597-605. [CrossRef]

21. Alberti, K.G.; Zimmet, P.; Shaw, J. Metabolic syndrome-A new world-wide definition. A Consensus Statement from the International Diabetes Federation. Diabet. Med. 2006, 23, 469-480. [CrossRef]

22. Armstrong, L.C.; Bornstein, P. Thrombospondins 1 and 2 function as inhibitors of angiogenesis. Matrix Biol. 2003, $22,63-71$. [CrossRef]

23. Guglielmi, V.; Cardellini, M.; Cinti, F.; Corgosinho, F.; Cardolini, I.; D'Adamo, M.; Zingaretti, M.C.; Bellia, A.; Lauro, D.; Gentileschi, P.; et al. Omental adipose tissue fibrosis and insulin resistance in severe obesity. Nutr. Diabetes 2015, 5, e175. [CrossRef]

24. Guzman-Ruiz, R.; Tercero-Alcazar, C.; Rabanal-Ruiz, Y.; Diaz-Ruiz, A.; El Bekay, R.; Rangel-Zuniga, O.A.; Navarro-Ruiz, M.C.; Molero, L.; Membrives, A.; Ruiz-Rabelo, J.F.; et al. Adipose tissue depot-specific intracellular and extracellular cues contributing to insulin resistance in obese individuals. FASEB J. 2020, 34, 7520-7539. [CrossRef]

25. Datta, R.; Podolsky, M.J.; Atabai, K. Fat fibrosis: Friend or foe? JCI Insight 2018, 3, e122289. [CrossRef] [PubMed]

26. Halberg, N.; Khan, T.; Trujillo, M.E.; Wernstedt-Asterholm, I.; Attie, A.D.; Sherwani, S.; Wang, Z.V.; Landskroner-Eiger, S.; Dineen, S.; Magalang, U.J.; et al. Hypoxia-Inducible factor 1alpha induces fibrosis and insulin resistance in white adipose tissue. Mol. Cell Biol. 2009, 29, 4467-4483. [CrossRef] [PubMed]

27. Zhang, J.; Chu, M. Differential roles of VEGF: Relevance to tissue fibrosis. J. Cell Biochem. 2019, 120, 10945-10951. [CrossRef] [PubMed]

28. Buechler, C.; Krautbauer, S.; Eisinger, K. Adipose tissue fibrosis. World J. Diabetes 2015, 6, 548-553. [CrossRef]

29. Pasarica, M.; Gowronska-Kozak, B.; Burk, D.; Remedios, I.; Hymel, D.; Gimble, J.; Ravussin, E.; Bray, G.A.; Smith, S.R. Adipose tissue collagen VI in obesity. J. Clin. Endocrinol. Metab. 2009, 94, 5155-5162. [CrossRef] [PubMed]

30. Spencer, M.; Yao-Borengasser, A.; Unal, R.; Rasouli, N.; Gurley, C.M.; Zhu, B.; Peterson, C.A.; Kern, P.A. Adipose tissue macrophages in insulin-resistant subjects are associated with collagen VI and fibrosis and demonstrate alternative activation. Am. J. Physiol. Endocrinol. Metab. 2010, 299, E1016-E1027. [CrossRef]

31. Abdennour, M.; Reggio, S.; Le Naour, G.; Liu, Y.; Poitou, C.; Aron-Wisnewsky, J.; Charlotte, F.; Bouillot, J.L.; Torcivia, A.; Sasso, M.; et al. Association of adipose tissue and liver fibrosis with tissue stiffness in morbid obesity: Links with diabetes and BMI loss after gastric bypass. J. Clin. Endocrinol. Metab. 2014, 99, 898-907. [CrossRef] [PubMed]

32. DeBari, M.K.; Abbott, R.D. Adipose tissue fibrosis: Mechanisms, models, and importance. Int. J. Mol. Sci. 2020, $21,6030$. [CrossRef]

33. Huang, A.; Lin, Y.S.; Kao, L.Z.; Chiou, Y.W.; Lee, G.H.; Lin, H.H.; Wu, C.H.; Chang, C.S.; Lee, K.T.; Hsueh, Y.Y.; et al. InflammationInduced macrophage lysyl oxidase in adipose stiffening and dysfunction in obesity. Clin. Transl. Med. 2021, 11, e543. [CrossRef]

34. Creemers, E.E.; Cleutjens, J.P.; Smits, J.F.; Daemen, M.J. Matrix metalloproteinase inhibition after myocardial infarction: A new approach to prevent heart failure? Circ. Res. 2001, 89, 201-210. [CrossRef] 
35. Karsdal, M.A.; Nielsen, S.H.; Leeming, D.J.; Langholm, L.L.; Nielsen, M.J.; Manon-Jensen, T.; Siebuhr, A.; Gudmann, N.S.; Ronnow, S.; Sand, J.M.; et al. The good and the bad collagens of fibrosis-Their role in signaling and organ function. Adv. Drug Deliv. Rev. 2017, 121, 43-56. [CrossRef]

36. Cabral-Pacheco, G.A.; Garza-Veloz, I.; Castruita-De la Rosa, C.; Ramirez-Acuna, J.M.; Perez-Romero, B.A.; Guerrero-Rodriguez, J.F.; Martinez-Avila, N.; Martinez-Fierro, M.L. The roles of matrix metalloproteinases and their inhibitors in human diseases. Int. J. Mol. Sci. 2020, 21, 9739. [CrossRef]

37. Chang, E.; Varghese, M.; Singer, K. Gender and sex differences in adipose tissue. Curr. Diab. Rep. 2018, 18, 69. [CrossRef] [PubMed]

38. Newell-Fugate, A.E. The role of sex steroids in white adipose tissue adipocyte function. Reproduction 2017, 153, R133-R149. [CrossRef] [PubMed]

39. Gabrielsson, B.G.; Olofsson, L.E.; Sjogren, A.; Jernas, M.; Elander, A.; Lonn, M.; Rudemo, M.; Carlsson, L.M. Evaluation of reference genes for studies of gene expression in human adipose tissue. Obes Res. 2005, 13, 649-652. [CrossRef]

40. Sun, K.; Park, J.; Gupta, O.T.; Holland, W.L.; Auerbach, P.; Zhang, N.; Goncalves Marangoni, R.; Nicoloro, S.M.; Czech, M.P.; Varga, J.; et al. Endotrophin triggers adipose tissue fibrosis and metabolic dysfunction. Nat. Commun. 2014, 5, 3485. [CrossRef]

41. Liu, Y.; Aron-Wisnewsky, J.; Marcelin, G.; Genser, L.; Le Naour, G.; Torcivia, A.; Bauvois, B.; Bouchet, S.; Pelloux, V.; Sasso, M.; et al. Accumulation and changes in composition of collagens in subcutaneous adipose tissue after bariatric surgery. J. Clin. Endocrinol. Metab. 2016, 101, 293-304. [CrossRef] [PubMed]

42. Rajan, M.R.; Sotak, M.; Barrenas, F.; Shen, T.; Borkowski, K.; Ashton, N.J.; Biorserud, C.; Lindahl, T.L.; Ramstrom, S.; Scholl, M.; et al. Comparative analysis of obesity-related cardiometabolic and renal biomarkers in human plasma and serum. Sci. Rep. 2019, 9, 15385. [CrossRef] [PubMed]

43. Vandesompele, J.; De Preter, K.; Pattyn, F.; Poppe, B.; Van Roy, N.; De Paepe, A.; Speleman, F. Accurate normalization of real-time quantitative RT-PCR data by geometric averaging of multiple internal control genes. Genome Biol. 2002, 3, research0034.1. [CrossRef] [PubMed] 